\title{
Perlecan and Dystroglycan act at the basal side of the Drosophila follicular epithelium to maintain epithelial organization
}

\author{
Martina Schneider ${ }^{1, *, \dagger}$, Ashraf A. Khalil ${ }^{1,2, \neq}$, John Poulton ${ }^{3, \neq}$, Casimiro Castillejo-Lopez ${ }^{1}$, Diane Egger-Adam ${ }^{4}$, \\ Andreas Wodarz ${ }^{5}$, Wu-Min Deng ${ }^{3}$ and Stefan Baumgartner ${ }^{1}$
}

Dystroglycan (Dg) is a widely expressed extracellular matrix (ECM) receptor required for muscle viability, synaptogenesis, basementmembrane formation and epithelial development. As an integral component of the Dystrophin-associated glycoprotein complex, Dg plays a central role in linking the ECM and the cytoskeleton. Disruption of this linkage in skeletal muscle leads to various types of muscular dystrophies. In epithelial cells, reduced expression of Dg is associated with increased invasiveness of cancer cells. We have previously shown that $\mathrm{Dg}$ is required for epithelial cell polarity in Drosophila, but the mechanisms of this polarizing activity and upstream/downstream components are largely unknown. Using the Drosophila follicle-cell epithelium (FCE) as a model system, we show that the ECM molecule Perlecan (Pcan) is required for maintenance of epithelial-cell polarity. Follicle cells that lack Pcan develop polarity defects similar to those of $D g$ mutant cells. Furthermore, Dg depends on Pcan but not on Laminin A for its localization in the basal-cell membrane, and the two proteins bind in vitro. Interestingly, the Dg form that interacts with Pcan in the FCE lacks the mucin-like domain, which is thought to be essential for Dg ligand binding activity. Finally, we describe two examples of how Dg promotes the differentiation of the basal membrane domain: (1) by recruiting/anchoring the cytoplasmic protein Dystrophin; and (2) by excluding the transmembrane protein Neurexin. We suggest that the interaction of Pcan and Dg at the basal side of the epithelium promotes basal membrane differentiation and is required for maintenance of cell polarity in the FCE.

KEY WORDS: Perlecan, Dystroglycan, Laminin, Dystrophin, Neurexin, Polarity, Epithelia, Oogenesis, Drosophila

\section{INTRODUCTION}

Dystroglycan (Dg) is a widely expressed extracellular matrix (ECM) receptor and is required for epithelial development, basementmembrane formation, muscle viability and synaptogenesis (Winder, 2001). In vertebrates, Dg is synthesized as a single polypeptide and post-translationally cleaved into the extracellular glycoprotein $\alpha \mathrm{Dg}$ and the transmembrane protein $\beta \mathrm{Dg}$ (Ibraghimov-Beskrovnaya et al., 1992). The two subunits are believed to remain attached to one another through non-covalent interaction of the C-terminal region of $\alpha \mathrm{Dg}$ with the N-terminal region of $\beta \mathrm{Dg}$ (Sciandra et al., 2001). $\alpha \mathrm{Dg}$ shows a dumbbell-like molecular shape in which two less glycosylated globular domains are separated by the mucin-like domain (mucin-domain), a highly glycosylated serine-threonineproline-rich region (Brancaccio et al., 1995). Laminin (Lam), Agrin, Perlecan (Pcan) and Neurexin (Nrx) serve as ligands for $\alpha \mathrm{Dg}$ (Ibraghimov-Beskrovnaya et al., 1992; Sugita et al., 2001), and Lam $\mathrm{G}$ (LG)-like domains mediate the interaction (Hohenester et al., 1999). The binding site on $\alpha \mathrm{Dg}$ is not known, but proper glycosylation of $\alpha \mathrm{Dg}$ is generally considered to be crucial for its

\footnotetext{
${ }^{1}$ Department for Experimental Medical Science, Section for Developmental Biology, Lund University, 22184 Lund, Sweden. ${ }^{2}$ Department of Protein Research, Genetic Engineering and Biotechnology Research Institute, Mubarak City for Scientific Research, Alexandria, Egypt. ${ }^{3}$ Department of Biological Science, Florida State University, Tallahassee, FL 32306-4370, USA. ${ }^{4}$ Fakultät für Biologie, Universität Konstanz, 78434 Konstanz, Germany. ${ }^{5}$ Department of Stem Cell Biology, DFG Research Center for Molecular Physiology of the Brain (CMPB), University of Göttingen, Justus-von-Liebig-Weg 11, 37077 Göttingen, Germany.

*Author for correspondence (e-mail: maschneider@bi.ku.dk)

†Present address: Department of Cell Biology and Comparative Zoology, University of Copenhagen, Universitetsparken 15, 2100 Copenhagen, Denmark

₹These authors contributed equally to this work
}

ligand-binding activity. Recent studies have demonstrated that Oglycosylation within the mucin-domain in required for Lam (Kanagawa et al., 2004) and Pcan binding (Kanagawa et al., 2005), but it is not clear whether the sugar-chains of this domain are directly involved in the interaction or merely play a structural role in supporting the rod-like shape of this region.

The cytoplasmic tail of $\beta D g$ interacts with Dystrophin (Dys) in muscle cells, and the Dys-homolog Utrophin (Utr) in epithelial cells. Dys/Utr in turn connect to actin filaments of the cytoskeleton. Dg therefore occupies a central position in an ECM-cytoskeleton link disruption of which leads to various types of muscular dystrophies (Cohn and Campbell, 2000). In addition, Dg has been suggested to play a key role in the transduction and modulation of various signaling cascades (Henry and Campbell, 1999; Winder, 2001).

In epithelial cells, reduced expression of $\mathrm{Dg}$ has been associated with increased invasiveness of cancer cells (Muschler et al., 2002). In some malignant tumors, e.g. prostate and mammary cancer, the expression of $\alpha \mathrm{Dg}$ is reduced (Henry et al., 2001; Muschler et al., 2002). Furthermore, the amount of reduction is correlated with the invasiveness of the tumor (Muschler et al., 2002). Recent results suggest that the loss of $\alpha \mathrm{Dg}$ might be an early event in carcinogenesis rather than being a consequence of neoplastic transformation (Sgambato and Brancaccio, 2005; Sgambato et al., 2003).

Some reports have suggested that the major ligand for Dg in nonmuscle cells might be Pcan, because the binding of $\alpha \mathrm{Dg}$ to Pcan LG-domains is five times stronger than that to the most active Lam fragment (Andac et al., 1999; Talts et al., 1999). Pcan is the major heparan sulfate proteoglycan in basement membranes (BMs) and connective tissue, and has been implicated in adhesion, proliferation, development and growth-factor binding (Iozzo, 1994). The Pcan core protein consists of five domains and binds to 
a variety of molecules, including FGF-7, Fibronectin, Heparin, Laminin 1, PDGF-B, $\alpha$ Dg and Integrins. At the $\mathrm{N}$-terminal domain I and the C-terminal domain V, glucosaminoglycan (GAG) chains are attached that interact with Laminin-1 and Collagen IV and bind to FGF-2, promoting its angiogenic and mitotic activities (Iozzo, 1994). Studies in transgenic mice have shown that Pcan is required for the maintenance of the functional and structural integrity of BMs in the heart, but is not needed for BM assembly per se (Costell et al., 1999).

Not much is known about the function of the interaction between Pcan and Dg. During the development of the neuromuscular junction, binding between Pcan and Dg is required for clustering of acetylcholine esterase at the postsynaptic membrane (Peng et al., 1999). In addition, cell culture studies with Pcan- and Laminin $\alpha 2$ deficient skin fibroblasts revealed that shedding of $\mathrm{Dg}$ is increased by the lack of Pcan, but not by lack of Laminin $\alpha 2$ (Herzog et al., 2004).

Pcan, Dg and other components of the Dystrophin-glycoprotein complex are conserved in Drosophila and vertebrates (Greener and Roberts, 2000; Voigt et al., 2002). Drosophila Pcan is encoded by terribly reduced optical lobes (trol) and is required for controlling proliferation of neuronal stem cells in the larval brain (Voigt et al., 2002). Pcan has been suggested to act in the ECM by binding, storing and sequestering external signals, including FGF and Hedgehog (Voigt et al., 2002). A role for Pcan in epithelial development has not been reported so far.

Drosophila Dg plays a role in polarizing epithelial cells and the oocyte (Deng et al., 2003). In particular, Dg function has been investigated during the development of the follicle-cell epithelium (FCE). The FCE forms through a mesenchymal-epithelial transition and uses mechanisms operating on the apical, lateral and basal side for epithelial differentiation (Tanentzapf et al., 2000). Contact of follicle cells with the basement membrane and with the germline cells has been suggested to play a role in polarizing the cells. As a result, distinct basal, apical and lateral cell-membrane domains are established by accumulating protein complexes that are actively reinforcing cell-membrane polarity. Loss of $\mathrm{Dg}$ leads to an expansion of apical markers to the basal side of the cells and loss of lateral markers. Some $D g$ mutant cells lose their epithelial appearance, form multiple layers and eventually die (Deng et al., 2003).

The finding that $\mathrm{Dg}$ is required for epithelial cell polarity is particularly interesting because of its role during the invasive behavior of cancer cells, but little is known about the molecular mechanism behind this polarizing activity.

In this study, we investigate the hypothesis that Pcan and Dg constitute a basal polarizing cue required for the differentiation of the basal membrane domain and epithelial cell polarity. We chose the FCE as a model system for several reasons: first, all follicle cells are derived from two to three somatic stem cells, making mosaic analysis an excellent tool with which to study gene function in epithelial development (Margolis and Spradling, 1995); second, the trol gene is transcribed in follicle cells (Voigt et al., 2002); and third, we have previously shown that Dg plays a role in follicle-cell polarization (Deng et al., 2003).

\section{MATERIALS AND METHODS} Fly stocks

Drosophila melanogaster stocks were raised on standard cornmeal-yeastagar medium at $25^{\circ} \mathrm{C}$ and are as follows: FRT101, pcan $^{\text {null }} / F M 7$ act-GFP (a gift from A. Voigt) (Voigt et al., 2002); FRT2A $\operatorname{lan} A^{9-32}$ (Deng and RuoholaBaker, 2000); (Henchcliffe et al., 1993), FRT42D D ${ }^{323}$ (Deng et al., 2003);
$D g$-hairpin $U A S$ - $d s D g$ (Deng et al., 2003); Dys-hairpin UAS-dsDys (the present study); $U A S-D g-C$ (Deng et al., 2003); $U A S-D g-B$ (this study); $a c t<F R T-C D 2-F R T<$ Gal4; UAS-GFP (Pignoni and Zipursky, 1997).

The following stocks were obtained from the Bloomington Stock Center: hsFLP, FRT2AGFP/TM3; FRT42D GFP/CyO; hsFLP, Sco/CyO, FRT101 GFP, MKRS hsFLP/TM6, act<FRT-CD2-FRT<Gal4, UAS-GFP.

\section{Generation of follicle cell clones}

Loss-of function mosaic and follicle-cell clones overexpressing UASconstructs were induced as previously described (Deng et al., 2003).

\section{Construction of the UAS-Dg-B construct}

$U A S-D g-B$ was constructed by cloning the $B g l \mathrm{II} / \mathrm{X} h o \mathrm{I}$ insert of the EST clone SD06707 into the pUAST transformation vector.

\section{Construction of the Dys-hairpin}

A 492 bp fragment common to the three transcripts of the Dys gene was amplified from cDNA with the primers CGGTACCTGATCGCTCAGTATTGCCAGGCT and AGGATCCGGGTCTGGAGGGTATTGGGT. After digestion with $K p n \mathrm{I} / \mathrm{BamHI}$, the fragment was cloned both into pBluescript II (Stratagene), forming the pKS-dys, and into pEGFP-N1 (Clontech), forming pEGFP-N1-dys. Inversion of the sequence was carried out by excision of the NheI/BamHI fragment of the pEGFP-N1-dys construct and subsequent ligation with a $148 \mathrm{bp} \mathrm{Sau3A}$ linker into pKs-Dys, cut with SpeI/BamHI. The1132 bp KpnI fragment containing the inverse sequence separated by the linker was inserted into pUAST. Before transformation the construct was verified by restriction analysis and sequencing.

\section{Glycoprotein extraction}

Glycoprotein was extracted at $4{ }^{\circ} \mathrm{C}$ in the presence of protease inhibitors according to a modification of the methods of Smalheiser and Kim (Smalheiser and Kim, 1995) and Collins (Collins et al., 2001). Drosophila embryos $(100 \mathrm{mg}$ ) were added to $1 \mathrm{ml}$ cold Tris-buffered saline [TBS, 25 $\mathrm{mM}$ Tris- $\mathrm{HCl}(\mathrm{pH} 7.4), 100 \mathrm{mM} \mathrm{NaCl}$ plus $1 \%$ triton-X100 and $4 \%$ protease inhibitors, homogenized and then incubated for 1 hour with rotation.

Wheat-germ agglutinin (WGA)-agarose (Vector Laboratories) were used to capture glycoproteins: $500 \mu \mathrm{l}$ WGA beads were incubated with the homogenate overnight, centrifuged and washed twice with TBS buffer containing $0.1 \%$ triton-X100 and $4 \%$ protease inhibitor (Roche Diagnostics). The WGA agarose was eluted twice with $250 \mu \mathrm{l}$ TBS buffer containing $0.1 \%$ triton-X100, $4 \%$ protease inhibitors and 0.3 $\mathrm{M} \mathrm{N}$-acetyl glucosamine (Sigma) under agitation for 10 minutes. The eluates were pooled, and then renatured by overnight dialysis in TBS buffer at $4^{\circ} \mathrm{C}$.

To remove non-specific binding components prior to immunoprecipitation experiments, we subjected the glycoprotein extract to a preclearing step using $5 \%(\mathrm{v} / \mathrm{v})$ rabbit serum for 1 hour, followed by 1 hour incubation with $5 \%(\mathrm{v} / \mathrm{v})$ of a $50 \%$ of protein A sepharose (PAS). The samples were centrifuged at $10,000 \mathrm{~g}$ for 10 minutes at $4^{\circ} \mathrm{C}$ and the supernatant collected in a new tube.

\section{Co-immunoprecipitation}

Lyophilized PcanV (Friedrich et al., 2000) was resuspended in deionized water to a final concentration of $10 \mu \mathrm{g} / \mu \mathrm{l}$ and stored at $-80^{\circ} \mathrm{C}$. Three concentrations of PcanV $(0.05,0.5$ and $5 \mu \mathrm{g} / \mu \mathrm{l})$ were prepared in total volumes of $450 \mu \mathrm{l}$ of binding buffer [ $25 \mathrm{mM}$ HEPES$\mathrm{NaOH}$ (pH 7.4), $100 \mathrm{mM} \mathrm{NaCl}, 1 \mathrm{mM} \mathrm{CaCl}_{2}, 1 \mathrm{mM} \mathrm{MnCl}_{2}, 2.5 \mathrm{mM}$ $\mathrm{MgCl}_{2}, 0.5 \mathrm{mM}$ PMSF]. Aliquots of $50 \mu \mathrm{l}$ of precleared glycoprotein lysate were added to the PcanV suspension and incubated overnight with gentle agitation.

Anti-Dystroglycan antibody (anti-Dg ${ }^{\text {pep}}$ ) was absorbed onto PAS beads to saturation by incubation overnight with gentle agitation. The beads were recovered by centrifugation at $10,000 \boldsymbol{g}$ for 2 minutes and washed thoroughly with TBS. Approximately $25 \mu \mathrm{l}$ of anti-Dg ${ }^{\mathrm{pep}}$-saturated PAS beads were added to $500 \mu \mathrm{l}$ of immune complexes and incubated overnight with gentle agitation. The beads were collected by centrifugation, at $10,000 \mathrm{~g}$ for 2 minutes, and washed three times with binding buffer. Controls of boiled anti$\mathrm{Dg}^{\mathrm{pep}}$, no anti-Dg ${ }^{\mathrm{pep}}$ and rabbit serum were included in all experiments. The 
precipitating proteins were split into two portions, resolved by SDSpolyacrylamide gel electrophoresis (SDS-PAGE) and subjected to immunoblotting with anti-Dg ${ }^{\text {cyto }}(1: 500)$ and anti-PcanV (1:500) according to standard procedures.

\section{Antibody production}

Rabbit anti-Dys antibodies were generated and affinity purified against peptide TSDTEANHDSDSRYM (amino acids 2366-2380; Eurogentec). Rabbit anti-Dgex8 (1:1000) was raised against the region corresponding to exon 8 (amino acids 243-507). Exon 8 was synthesized by PCR with primers ACGAATTCTTGGAGGTGTCG and GGTCTAGATTATGGCGATTCAGACAGTG (DNA Technology), and LD11619 as template. The PCR product was digested with $E c o$ RI and $X b a \mathrm{I}$, and cloned into vector pMALc2. The purified fusion protein was used to raise a polyclonal antiserum (Antibody AB), which was affinity purified with the fusion protein. Rabbit anti-Dg ${ }^{\text {pep }}$ antibodies were generated and affinity purified against peptide GKSPATPSYRKPPPYVSP (Neosystems)

\section{Histochemistry}

Ovary staining was performed as described previously (Deng et al., 2001). The following antibodies were used: rabbit anti-Dys (1:1000), rabbit anti$\operatorname{Dg}^{\text {ex8 }}$ (1:1000), rabbit anti-Dg ${ }^{\text {cyto }}$ (1:1000) (Deng et al., 2003), rabbit antiLaminin 329 (1:1000) (Gutzeit et al., 1991) (Fig. 4I), rabbit anti-Laminin $\alpha$ (1:1000) (Kumagai et al., 1997) (a gift from Y. Kitagawa) (Fig. 4H,J,K), rabbit anti-Bazooka (1:500) (Wodarz et al., 2000), rabbit anti-PcanV (1:1000) (Friedrich et al., 2000), mouse anti-Armadillo (1:40) (Hybridoma Bank), mouse anti-Crb Cq4 (1:25) (Hybridoma bank), rabbit anti-Discslarge (1:1000) (Woods and Bryant, 1991), mouse anti- $\beta 1 P S$ Integrin CF.6G11 (1:1000) (a gift from Danny Brower), rabbit anti-NrxIV (1:1000) (Baumgartner et al., 1996), mouse anti-Nrg 1B7 (1:500) (Bieber et al., 1989) (a gift from M. Hortsch), guinea pig anti-Cont [1:2000 (Faivre-Sarrailh et al., 2004); a gift from Manzoor Bhat], anti-rabbit IgG-FITC (DAKO), antimouse IgG-Cy3 (Amersham) and Alexa 568 Phalloidin (Molecular Probes). Nuclei were stained with TO-PRO (1:200, Molecular Probes) or DAPI (10 $\mu \mathrm{g} / \mathrm{ml})$. Confocal pictures were taken with a Leica TCS SP2 and processed with Adobe Photoshop.

\section{Prediction of glycosylation sites}

Potential mucin type GalNAc O-glycosylation sites were predicted with the NetOGlyc 3.1 server (Julenius et al., 2005).

\section{RESULTS \\ Perlecan is required for integrity of the follicle cell epithelium}

The FCE has a typical apicobasal polarity; the apical side faces the germ cells, and the basal side faces the basement membrane (Fig. 1A). Pcan can be detected around stage 1-2 of oogenesis in vesiclelike dots inside or between the follicle cells, and in the basal lamina after stage 3 (Fig. 1B). As development proceeds, Pcan accumulates in the BM, and the dot-like staining disappears (Fig. 1B). Similar to Lam, Pcan can be detected in stripes that run perpendicular to the anteroposterior axis of the egg (Fig. 1C). A strong expression of Pcan can also be seen in the muscular sheath surrounding the ovariole (Fig. 1B). To determine whether Pcan is required for FCE development, we induced somatic follicle-cell clones homozygous for the trol loss-of-function allele rrol $^{\text {null }}$. trol $^{\text {null }}$ completely and exclusively lacks the Pcan-coding region (Voigt et al., 2002). In a clone of homozygous trol ${ }^{\text {null }}$ cells, hereafter called trol clone, the dot-like Pcan staining is completely absent (Fig. 1D). Small trol clones that were induced during later stages still show Pcan protein localization in the BM (data not shown). trol clones frequently loose epithelial tissue organization. In egg chambers of stage 6-9, about $50 \%$ of the clones displayed a multilayer phenotype ( $n=44$, Fig. 1E). In addition, mutant follicle cells were aberrantly placed between the germ cells (Fig. 1E). These results clearly show that Pcan is required for the integrity of the FCE.

\section{Perlecan is required for maintaining cell membrane polarity}

To determine whether the breakdown of epithelial organization in trol clones is caused by a defect in cell polarity, we analyzed the distribution of a collection of cell-polarity markers. Bazooka (Baz), a component of the Baz-complex, is normally expressed in the apical membrane domain. In trol clones, Baz is generally enriched and expanded to the cytoplasm (Fig. 2A,B). Crumbs (Crb) and Patj are components of the Crb complex, which is expressed apically. Although Patj localization is not changed in trol clones (Fig. 2C),
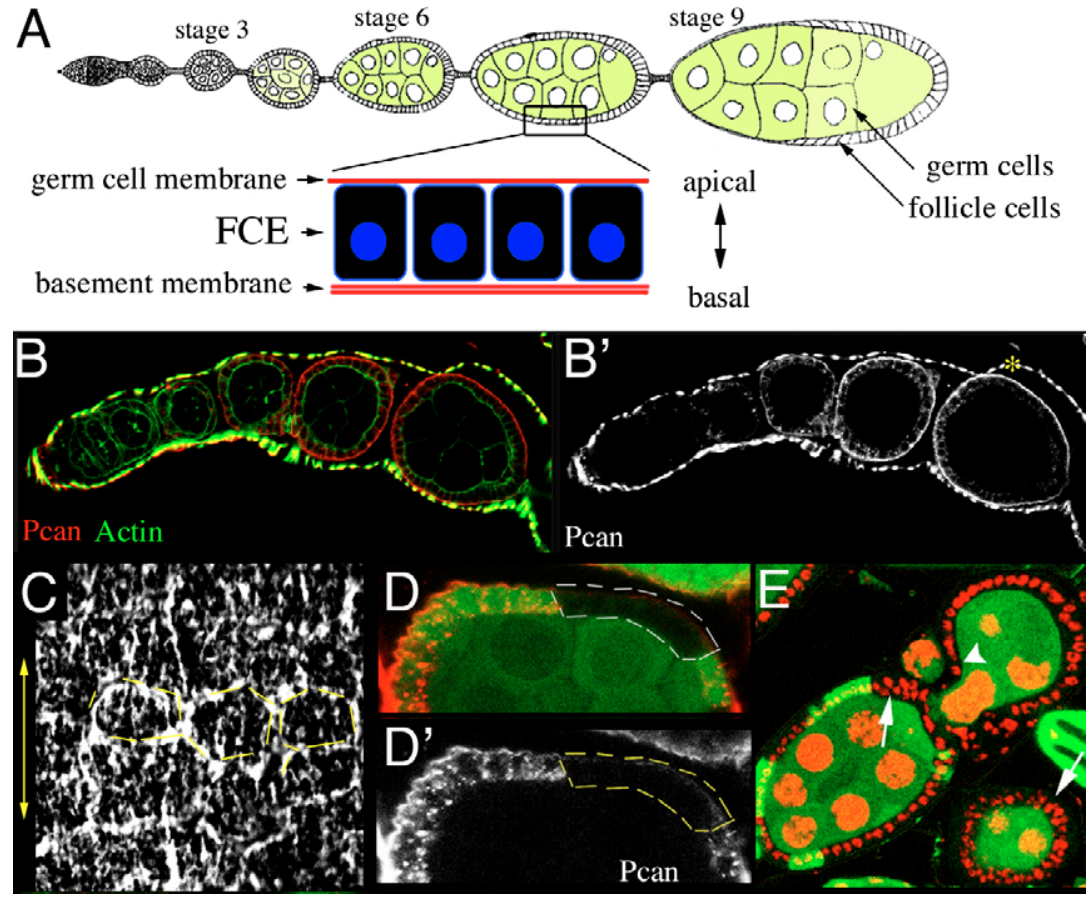

Fig. 1. Perlecan is required for integrity of the follicle cell epithelium. (A) Schematic drawing of an ovariole and FCE. (B, $\left.\mathbf{B}^{\prime}\right)$ Pcan accumulates in the $\mathrm{BM}$ after the FCE is formed and is expressed in the muscular sheath (asterisk). (C) Pcan stripes run perpendicular to the long axis of the egg chamber. Yellow lines indicate cell borders. The arrow indicates the orientation of the stripes. $\left(\mathbf{D}, \mathbf{D}^{\prime}\right)$ In a trol clone (broken line) expression of Pcan is absent. Red, Pcan; green, GFP. (E) trol clones frequently loose their epithelial organization and become multilayered (arrows). Occasionally mutant cells can be found in aberrantly positioned between the germ cells (arrowhead). Green, GFP; red in E, DNA Clones are marked by the absence of GFP. 
Crb staining is frequently reduced (Fig. 2D). Armadillo/ $\beta$-catenin (Arm) is a cytoplasmic component of the adherens junctions located at the apical side of the lateral membrane. Arm expression appeared to be slightly elevated and expanded to all cell membranes (Fig. 2E,F). Discs-large (Dlg) is normally localized at the lateral
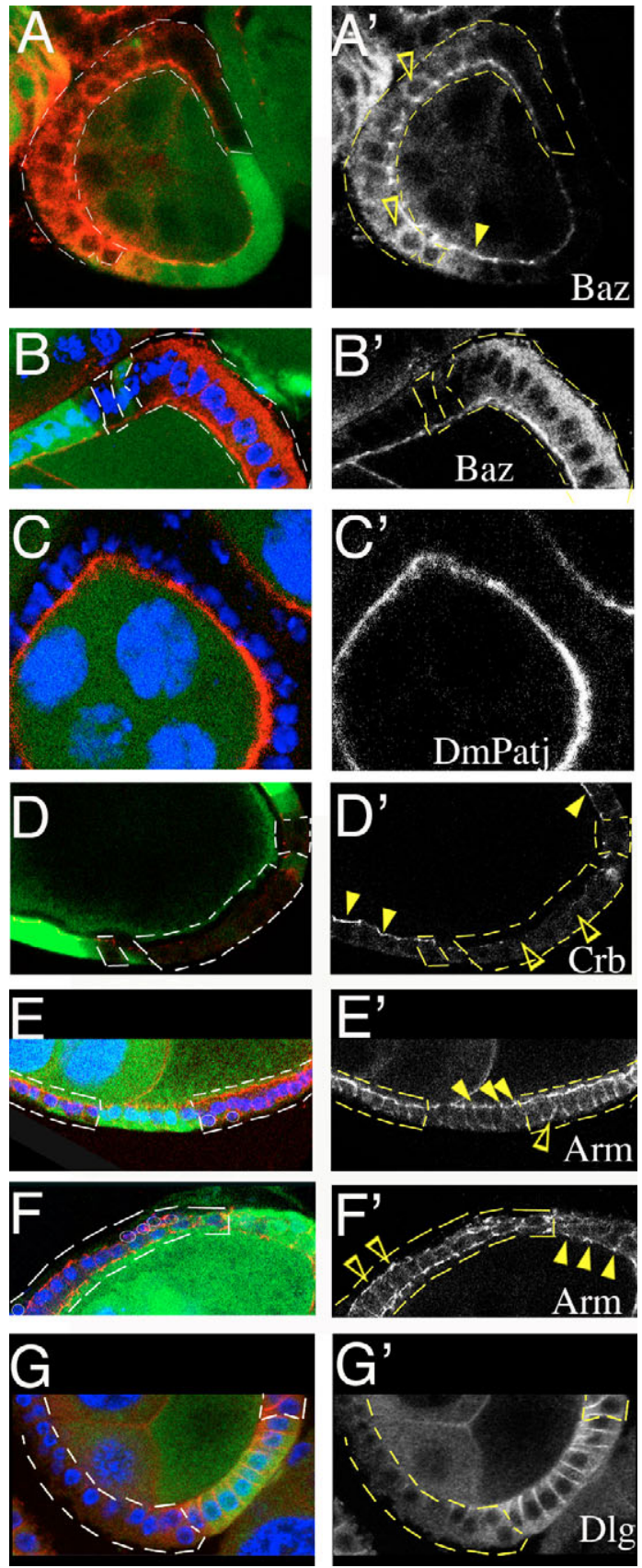

Fig. 2. Perlecan is required for cell membrane polarity. tro/ clones show defects in the distribution of various cell polarity markers (filled arrowheads indicate normal; open arrowheads disrupted protein localization). (A,B) Baz (red) is generally enriched and expanded into the cytoplasm. (C) Patj (red) is not affected by the loss of Pcan.

(D) Apical localization of $\mathbf{C r b}$ (red) is frequently reduced. (E,F) Arm (red) expression is slightly elevated and expanded to all cell membrane.

(G) Dlg (red) is strongly reduced. A'-G' show red channels only. Clones are marked by the loss of GFP and outlined by broken lines. Nuclei of follicle cells in ectopic positions are marked with a circle. Green, GFP; blue, DNA. membrane domain as part of the Dlg complex. In trol clones, Dlg staining is strongly reduced (Fig. 2G). These results demonstrate that loss of Pcan leads to cell-polarity defects.

\section{Perlecan domain V can bind to Dystroglycan in vitro}

The polarity defects observed in trol clones are reminiscent of those caused by the lack of Dg (Deng et al., 2003). Pcan and Dg interact as ligand and receptor in vertebrates, which prompted us to investigate whether this interaction is conserved in the fly. As domain $\mathrm{V}$ of mouse Pcan has been shown to be a high-affinity ligand for $\alpha \mathrm{Dg}$ (Talts et al., 1999), we performed a co-immunoprecipitation experiment using recombinantly expressed Drosophila Pcan domain $\mathrm{V}$ (PcanV) and embryonic protein extract enriched for glycoproteins. Different amounts of purified PcanV were added to the glycoprotein extract and immunoprecipitation was performed with an antibody directed against the $\mathrm{C}$ terminus of $\mathrm{Dg}$ ( (anti-Dgep). Western blot analysis showed that in addition to Dg, PcanV was present in the precipitate (Fig. 3). No co-immunoprecipitation of PcanV occurred in the absence of glycoprotein extract, with boiled Dg-antibody or with rabbit serum (Fig. 3). The experiment was repeated twice, with similar results, with different glycoprotein preparations (data not shown). Although we have previously used anti-Do $^{\text {pep }}$ to immunoprecipitate all major forms of Dg (data not shown) only a $\sim 120 \mathrm{kDa}$ band was detected in the precipitate which might reflect the abundance of this form (compare with Fig. 5C).

\section{Perlecan is required for Dystroglycan localization}

We next investigated the effect of Pcan on the distribution of Dg and vice versa. Using an antibody directed against the cytoplasmic domain of $\mathrm{Dg}$, anti-Dg ${ }^{\text {cyto }}$, we found that in trol clones, $\mathrm{Dg}$ is frequently lost from the basal cell membrane (Fig. 4A). Some variability in this phenotype was observed, which probably reflected perdurance of Pcan in the ECM (data not shown). Occasionally, apical expression of Dg seemed to be increased in the clone (Fig. 4B). Pcan, however, was normally localized in $D g$ clones (Fig. 4C). A disruption of the filamentous organization was not obvious (Fig. 4D).

To investigate whether Pcan is required specifically for localization of $\mathrm{Dg}$, or also affects other ECM receptors, we determined Integrin (Int) localization in trol clones using antibodies

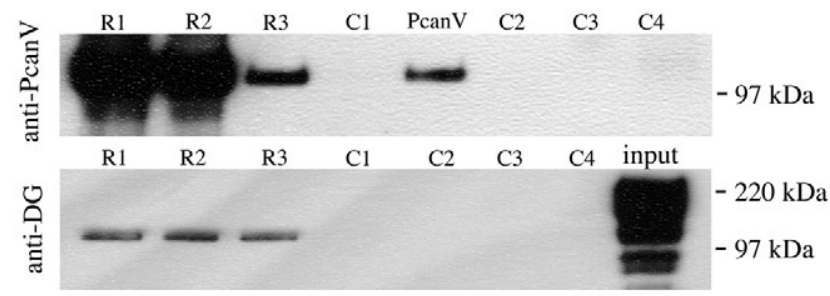

Fig. 3. Dystroglycan and Perlecan can bind in vitro. Western blots showing co-immunoprecipitation of PcanV with anti-Dg ${ }^{\text {pep }}$. Embryonic glycoprotein extract was mixed with $5 \mu \mathrm{g}$ PcanV (R1), $0.5 \mu \mathrm{g}$ PcanV (R2) or $0.05 \mu \mathrm{g}$ PcanV (R3), and precipitated with anti-Dg ${ }^{\text {pep }}$. Controls were: no glycoprotein extract added to reaction mixture (C1), precipitation reaction with boiled $\mathrm{Dg}$ antibody (C2), only primary antibody loaded (C3), primary antibody exchanged for rabbit serum (C4). The precipitates were probed with anti-PcanV (upper panel) or anti-Dg cyto (lower panel). Pure PcanV (PcanV $0.05 \mu \mathrm{g}$ ) and glycoprotein extract (input) were loaded as a positive control. Some of the minor Dg bands in the glycoprotein extract (input) are probably due to protein degradation. 


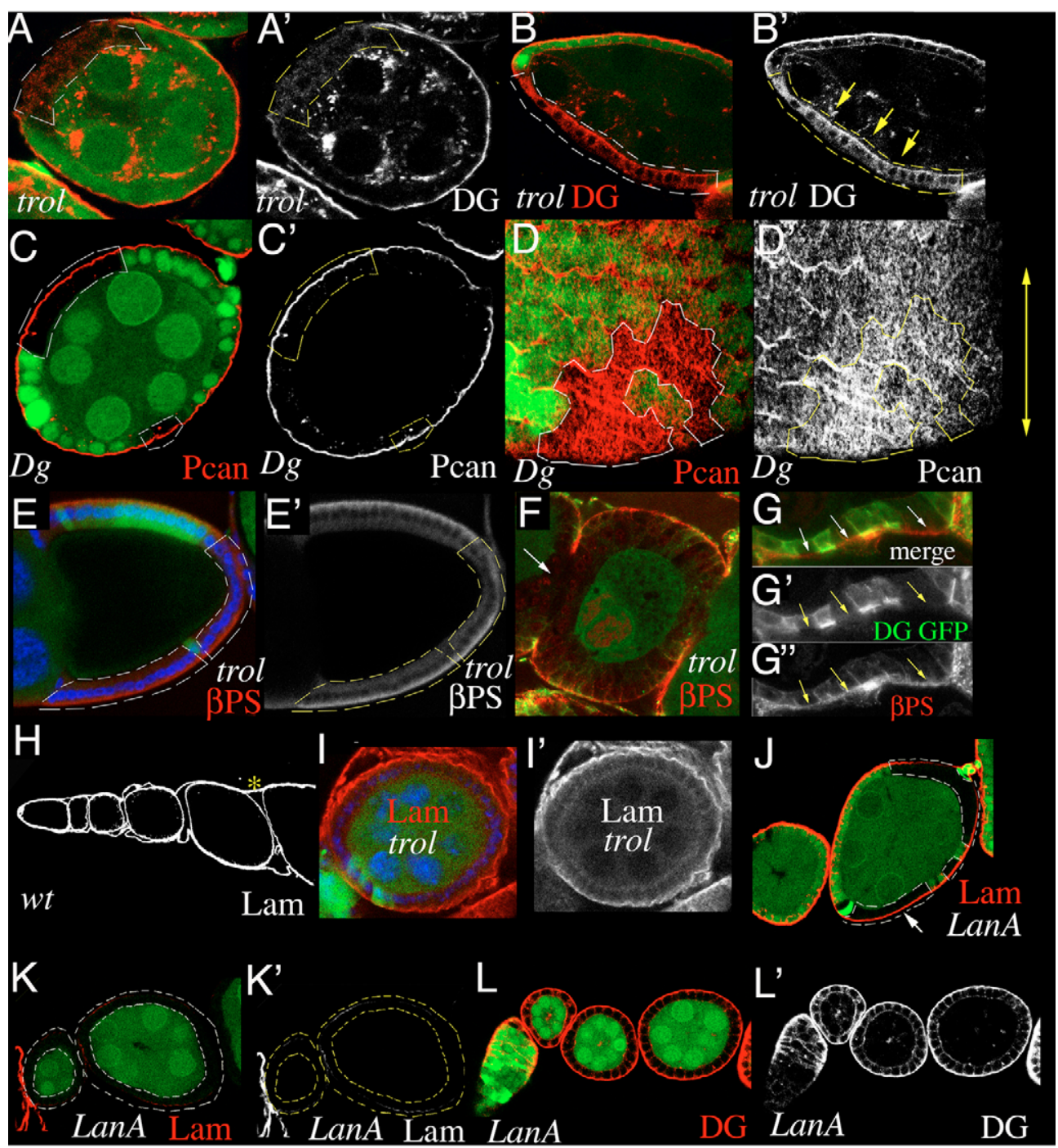

Fig. 4. Perlecan is required for basal localization of Dystroglycan. (A,B) Dg (red) is no

longer present in the basal cell membrane of trol clones.

Occasionally, Dg appears to be redistributed to the apical cell membrane (yellow arrows in $\mathrm{B}^{\prime}$ ).

(C,D) Pcan localization in the ECM is unchanged in a $\mathrm{Dg}^{323}$ clone (C) and orientation of Pcan-stripes appears normal (D). (E) $\beta P S$ Int (red) is normally expressed in trol clones. Blue indicates DNA. (F) In a FCE entirely composed of mutant cells, gaps in $\beta P S$ staining can be seen. Green indicates GFP and Dg; arrow indicates gap. (G) $\beta P S$ Int (red) is still present (arrows) in the basal membrane of a trol clone that has lost Dg (green). (G') Green channel only, showing both GFP and antiDg cyto staining. (G") Red channel only. (H) Expression of Lam in a wild-type ovariole. Asterisk indicates muscular sheath. (I) A very large trol clone showing fuzzy Lam (red) distribution. Blue indicates DNA. (J) Lam (red) is still present at the basal side of a medium sized lanA clone (arrow). (K) Two egg chambers whose FCE are entirely composed of lanA mutant cells. Lam (red) is almost completely absent. (L) Three egg chambers whose FCE are entirely composed of lanA mutant cells. Dg (red) is still localized at the basal membrane. Clones are marked by the loss of GFP (green) and indicated by broken lines $\left(A^{\prime}-L^{\prime}\right)$ Red channels only.

directed against the Drosophila $\beta$ subunit of Int, $\beta$ PS. $\beta$ PS was not significantly reduced in trol clones (Fig. 4E). Only in an FCE entirely composed of mutant cells a disruption of $\beta$ PS staining was occasionally observed (Fig. 4F). To compare $\beta P S$ and Dg expression in trol clones directly, we performed a Dg and $\beta P S$ double staining. Dg was frequently lost from the basal membrane even in smaller trol clones, while $\beta$ PS expression appeared unchanged (Fig. 4G).

A possible explanation for the alteration of $\mathrm{Dg}$ localization in trol cells is that Pcan influences localization of Dg indirectly through other Dg ligands. Although analysis of perlecan knockout mice demonstrated that Pcan is dispensable for basement membrane formation in general (Costell et al., 1999), studies in mouse embryonic stem cells suggested a role for Pcan in organizing Lam in the ECM (Henry et al., 2001). Furthermore, recent findings suggested a trimolecular complex of Pcan, Lam and Dg (Kanagawa et al., 2005). We therefore determined Lam distribution in trol clones. Lam is expressed at the basal side of the FCE and in the muscular sheath (Fig. 4H). Even in very large trol clones, Lam is clearly present; however, the staining appeared to be diffuse relative to the wild type (Fig. 4I). To test directly whether Lam is required for Dg localization, we induced clones homozygous for $\operatorname{lan} A^{9-32}$, a null allele of lanA (Henchcliffe et al., 1993). The lanA gene codes one of the two Drosophila $\alpha$-chains. The second $\alpha$-chain (Wing blister) is not expressed in the FCE with exception of the border cells (data not shown). Although medium-sized lanA clones did seldom show reduction of Lam (Fig. 4J), Lam staining was almost completely abolished when the entirely FCE was composed of lanA mutant cells (Fig. 4K). In these 'all-mutant-FCE', Dg was virtually unaffected (Fig. 4L), indicating that Lam is not required for $\mathrm{Dg}$ localization. Similarly, Pcan expression appeared normal despite the lack of LanA showing only small regions of reduced staining (data not shown).

We conclude that Dg depends on Pcan for localization in the basal membrane domain. Our results further suggest that this dependency is specific for Pcan and Dg.

\section{Perlecan-dependent Dystroglycan lacks the mucin- like domain}

Glycosylation of Dg is widely accepted to be essential for its function, and recent results suggest an important role for $\mathrm{O}$ glycosylation in the mucin-like domain for binding to Lam (Kanagawa et al., 2004) and Pcan (Kanagawa et al., 2005). In this context, it is interesting that Drosophila $D g$, unlike vertebrate $D g$, is subjected to alternative splicing which results in at least two shorter forms of $\mathrm{Dg}$ that lack the putative mucin-like domain (Deng et al., $2003)$. In addition to the longest $D g$-mRNA $(D g-C)$, which codes for 

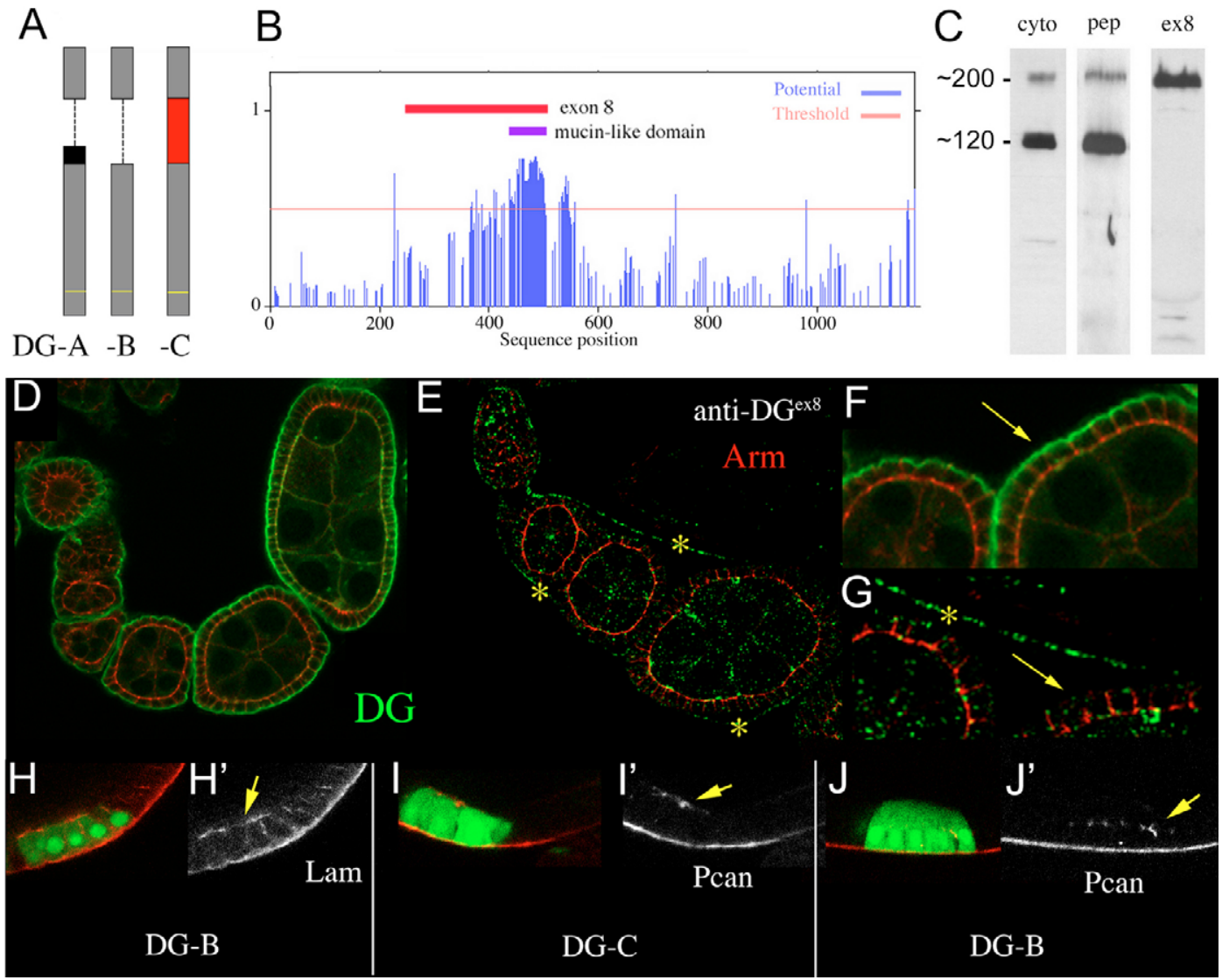

Fig. 5. Perlecan-dependent Dystroglycan lacks the mucin domain. (A) Schematic drawing of the Dg forms $A, B$ and $C$, which are generated through differential splicing of exon 8 (red box) and exon 9 (black box). The transmembrane-domain is indicated with a yellow line. (B) Graphic showing the potential of mucin type O- linked glycosylation for each position of the Dg-C sequence. A stretch of 80 amino acids (positions $424-$ 503 , indicated by the blue bar) contains a cluster of 52 high potential o-glycosylation sites, which constitute the mucin-like domain. The red bar indicates the region encoded by exon 8 (position 243-507). (C) Western blots of embryonic protein extract (0-20 hours) probed with anti-Dgcyto (cyto), anti-Dg ${ }^{\text {pep }}$ (pep) and anti-Dg ${ }^{\text {ex8 }}$ (ex8). (D-F) Wild-type ovaries stained with anti-Dg ${ }^{\text {cyto }}(\mathrm{D}, \mathrm{F})$ and anti-Dg ${ }^{\text {ex8 }}$ (E,G). F and G are higher magnification of $D$ and $E$, respectively. $D g$ is strongly concentrated in the basal membrane of the FCE throughout oogenesis ( $D$, arrow in $F)$. Dg-C is expressed in the muscular sheath (yellow asterisks in E and G) but absent from the basal membrane of the FCE (arrow in G). Red, Arm; green, Dg. $(\mathbf{H}-\mathbf{J})$ Ectopic expression of Dg-B $(\mathrm{H}, \mathrm{J})$ and Dg-C (I) induces ectopic accumulation (arrows) of Lam $(\mathrm{H})$ and Pcan $(\mathrm{I}, \mathrm{J})$. Cells expressing the Dg construct are marked by GFP (green). Red indicates Lam (H) and Pcan $(I, J)$. ( $\left.\mathrm{H}^{\prime}-J^{\prime}\right)$ Red channels only.

a protein $(\mathrm{Dg}-\mathrm{C})$ with a calculated molecular weight of $130 \mathrm{kDa}$, two shorter mRNAs ( $D g-A$ and $D g-B)$ are generated that code for proteins with calculated molecular weight of $111 \mathrm{kDa}(\mathrm{Dg}-\mathrm{A})$ and $105 \mathrm{kDa}$ (Dg-B) (Fig. 5A). Both shorter mRNAs lack exon 8, which contains the coding sequences for the putative mucin-domain consisting of 52 potential O-linked glycosylation sites that are clustered within a stretch of 80 amino acids (Fig. 5B).

In our hands, western blotting of embryonic protein extracts with anti-Dg antibodies reveals two major bands of $\sim 120 \mathrm{kDa}$ and $~ 200$ $\mathrm{kDa}$. Both bands are recognized by two different antibodies directed against the cytoplasmic domain of $\mathrm{Dg}$ (anti-Dg ${ }^{\text {cyto }}$ and anti-Dg ${ }^{\text {pep }}$ ) (Fig. 5C). The high discrepancy between predicted (105, 111, 130 $\mathrm{kDa})$ and the observed molecular weight $(200 \mathrm{kDa})$ suggests that at least one form of $\mathrm{Dg}$ is subjected to substantial posttranslational modifications. To test whether these modifications take place within the mucin domain, we used an antibody specific for the region encoded by exon 8 (anti- $\mathrm{Dg}^{\mathrm{ex} 8}$ ). Anti-Dg ${ }^{\mathrm{ex} 8}$ recognized only the $\sim 200 \mathrm{kDa}$ band (Fig. 5C), suggesting that the $\sim 200 \mathrm{kDa}$ band represents Dg-C, while the $\sim 120 \mathrm{kDa}$ band, represents both $\mathrm{Dg}-\mathrm{A}$ and/or Dg-B. Taken together, our findings suggest that Dg-C contains a mucin domain, which is lacking in Dg-A and Dg-B.
To determine which of the Dg forms is actually expressed in the FCE, we compared the staining pattern of anti-Dg ${ }^{\text {cyto }}$ and anti-Dg ${ }^{\text {ex8 }}$. Staining with anti-Dg ${ }^{\text {cyto }}$ showed that $\mathrm{Dg}$ is highly concentrated in the basal membrane of follicle cells throughout oogenesis (Fig. $5 \mathrm{D}, \mathrm{F})$ and in the muscular sheath surrounding the egg chambers (data not shown). Staining with anti-Dg ${ }^{\text {ex8 }}$, however, revealed that the mucin-domain containing form $\mathrm{Dg}-\mathrm{C}$ is expressed in the muscular sheath surrounding the egg chamber, but is absent from the basal membrane of the follicle cells (Fig. 5E,G). The dot-like staining present in the FCE is unspecific staining because it still can be observed in a $D g$ follicle cell clone (data not shown).

To directly test the role of the mucin-domain for the interaction with Pcan and Lam, we ectopically expressed Dg-C and Dg-B in the FCE. As previously described, overexpression of $\mathrm{Dg}-\mathrm{C}$ induced ectopic accumulation of Lam (Deng et al., 2003; data not shown). We found that Dg-B, which differs from Dg-C only by the lack of exon 8 , was equally able to induce Lam accumulation (Fig. 5H). Furthermore, both forms induced ectopic accumulations of Pcan (Fig. 5I,J).

Taken together, our results suggest that interaction between $\mathrm{Dg}$ and Pcan (and possibly Lam) does not require the mucin-like domain of Dg. 


\section{Dystroglycan is required for localization of Dystrophin and vice versa}

Determining which molecular mechanisms Dg employs to maintain cell-membrane polarity requires knowledge about proteins that interact with the cytoplasmic domain of Dg. Two prime candidates are Dys and Utr, which are both represented by the single Dystrophin $(D y s)$ gene in the fly (Greener and Roberts, 2000). The C-terminal Dys-binding site in the cytoplasmic domain of Dg is well conserved in the fly (Greener and Roberts, 2000). To determine whether the interaction between Dg and Dys is conserved as well, we induced $D g$ follicle-cell clones and tested whether removal of Dg affected the intracellular localization of Dys. Whereas in wild-type follicle cells, Dys is localized at the basal membrane (Fig. 6A), in $D g$ mutant cells, the basal Dys staining was clearly reduced (Fig. 6B).

To test whether Dys is required for Dg localization, we used a Dys-hairpin construct, $d s D y s$, to reduce levels of the Dys protein in follicle cells (Fig. 6C). The reduction of Dys led to a reduction of Dg in the basal membrane (Fig. 6D). In trol clones, a diffuse cytoplasmic distribution of Dys was observed in instead of the basal localization (Fig. 6E), which is in agreement with our previous observation that Pcan is required for Dg localization. These results suggest that the interaction between Dg and Dys is likely to be conserved in the fly and that Dg plays a role in the differentiation of the basal membrane by recruiting and/or anchoring Dys.

\section{Dystroglycan is required for Neurexin and Contactin, but not for Neuroglian localization}

Previous studies suggested that the cell-surface receptor Neurexin (Nrx) binds Dg in the brain (Sugita et al., 2001), prompting us to determine whether the Drosophila homolog Neurexin IV (Baumgartner et al., 1996) is a likely candidate for binding to Dg and whether NrxIV is a specific membrane-polarity marker in follicle cells. In Drosophila, Nrx IV has been shown to be required for the formation of septate junctions (SJ) in epithelia and glia (Baumgartner et al., 1996). Epithelial SJ are located towards the apical end of the lateral membrane, basal to the adherens junction. In the FCE, incipient SJ have been observed at stage 6 that have matured into pleated SJ by stage 10 (Muller, 2000). To our knowledge, expression of NrxIV in the developing follicular epithelium has not been reported so far. We found that NrxIV is expressed in a dynamic pattern throughout oogenesis in both germline and follicle cells (Fig. 7A). During early stages, NrxIV appears to be unevenly distributed in the cytoplasm of follicle and germline cells. As oogenesis proceeds, NrxIV disappears from the cytoplasm of both cell types. In the follicle cells, NrxIV accumulates first at the basal side and then gradually disappears from the basal membrane and accumulates at the basal side of the lateral membrane (Fig. 7B). During SJ development, NrxIV forms a complex with Neuroglian (Nrg) and Contactin (Cont), and all three proteins are interdependent for SJ localization (Faivre-Sarrailh et al., 2004). To determine whether a similar complex exists in the follicle cell epithelium, we performed a triple staining.

Cont was weakly expressed during early stages of oogenesis (Fig. 7C). A dot-like staining was observed that appeared to be associated with the cell membrane (Fig. 7D). At later stages of oogenesis, Cont expression accumulated at the apical side of the lateral membrane (Fig. 7E). As described earlier (Wei et al., 2004), Nrg was expressed at the lateral membrane (Fig. 7F). All three proteins co-localized in dot-like structures, which appear to be associated with tripartite cell junctions (Fig. 7G).
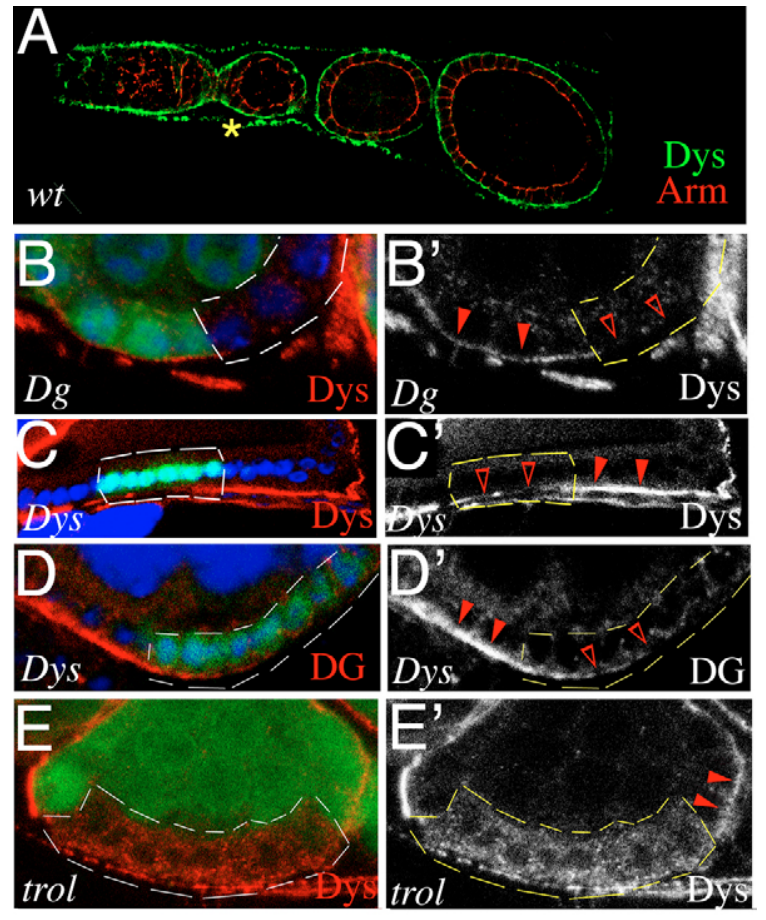

Fig. 6. Dystroglycan and Dsytrophin are mutually dependent for basal membrane localization. (A) In a wild-type ovariole, Dys is expressed in the muscular sheath (star) and at the basal membrane of the follicle cells. (B) In a $D g^{323}$ follicle cell clone, Dys (red) is lost from the basal membrane. (C) Expression of dsDys (Dys-hairpin) efficiently reduces Dys expression (red). (D) Reduction of Dys leads to a

reduction of $\mathrm{Dg}$ (red) in the basal membrane. (E) In a tro/ clone, Dys is no longer concentrated at the basal membrane but diffusely localized in the cytoplasm. All follicle cell clones are marked with broken lines. Filled arrowheads indicate the wild type; open arrows indicate the mutant expression pattern. ( $\left.B^{\prime}-E^{\prime}\right)$ Red channels only. Blue indicates DNA.

In $D g$ clones, NrxIV was no longer restricted basolaterally but was expressed throughout the basal membrane domain (Fig. 8A,B). A similar phenotype was observed in trol clones (Fig. $8 \mathrm{C}, \mathrm{D})$. Cont was found to colocalize with the ectopic accumulations of Nrx in $D g$ clones (Fig. 8E,F), whereas no change in Nrg localization was observed (Fig. 8E,F). These results suggest that Dg is required to exclude NrxIV and Cont from the basal membrane domain.

\section{DISCUSSION}

Cell-matrix interactions are crucial for epithelial morphogenesis and tissue organization, and many studies have demonstrated the importance of Laminin and its cell receptors in this context ( $\mathrm{Li}$ et al., 2003). So far as we know, our study is the first to show that Pcan plays an important role in epithelial polarization and tissue architecture, as well. The phenotypes caused by the loss of Dg or Pcan share many similarities, such loss of cell polarity, formation of multilayers and 'invasion' by mutant follicle cells of the spaces between germ cells. One interesting difference is the behavior of the apical marker Patj, which accumulated at the basal membrane in $D g$ clones (Deng et al., 2003), but was unaffected in trol clones (Fig. 2C). The reason for this difference is not known, but a possible explanation is that in trol mutant cells, $\mathrm{Dg}$ is still present and occasionally even enriched apically (Fig. 4B). 


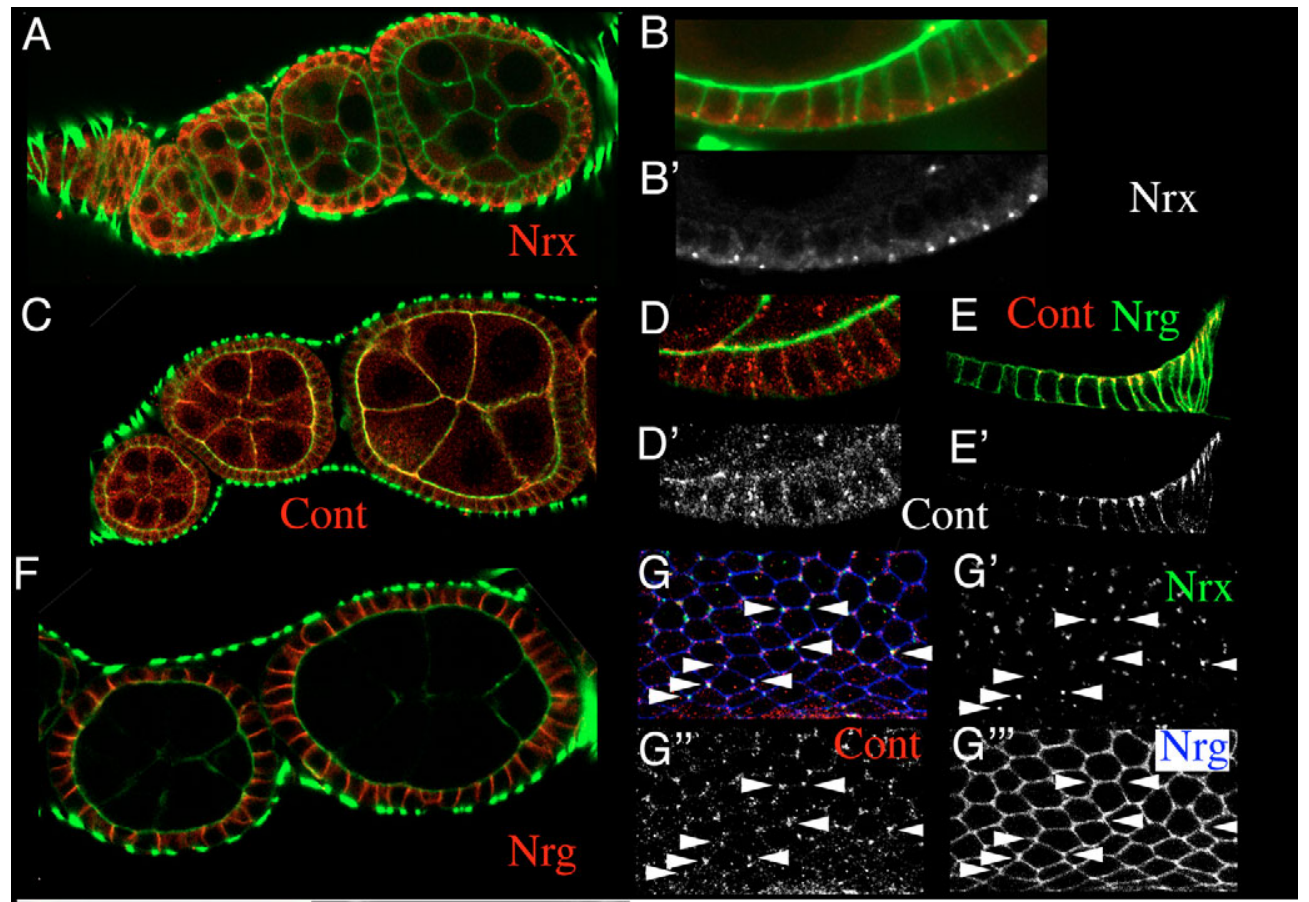

Fig. 7. Distribution of NrxIV, Con and Nrg in the FCE. All pictures show antibody staining of wildtype ovaries. (A,B) NrxIV (red) gradually concentrates at the basal side of the lateral membrane of follicle cells during development. (C-E) Cont (red) can bee seen in a punctate staining during early and mid-oogenesis (D). In older egg chambers (E), Cont concentrates apicolaterally. (F) $\mathrm{Nrg}$ (red) is expressed in the lateral cell membrane. (G) Surface views of the FCE showing NrxIV (green, $G^{\prime}$ ), Cont (red, $\mathrm{G}^{\prime \prime}$ ) and Nrg (blue, G'"). All three proteins co-localize at tricell junctions. Green indicates actin in $A-D, F .\left(B^{\prime}, D^{\prime}, E^{\prime}\right)$ Red channel only.

Patj is a cytoplasmic PDZ domain protein that forms an apical complex with the transmembrane protein Crb (Klebes and Knust, 2000). In contrast to Patj, Crb is frequently reduced in trol clones (Fig. 2D). A similar loss of Crb was observed in embryonic salivary gland after ectopic expression of Dg (Deng et al., 2003), suggesting that the apical enrichment of Dg in trol clones might cause the reduction of Crb. Furthermore, our results confirm the existence of a Crb-independent localization and retention mechanism for Patj in the FCE that has been suggested earlier (Tanentzapf et al., 2000).

Another difference between trol and $D g$ clones lies is the ability of the cells to survive.. Whereas $D g$ clones eventually die, trol clones can survive until later stages of oogenesis (data not shown). Studies of embryoid bodies deficient in Dg revealed an accelerated level of apoptosis, which has led to the proposal that Dg has a role in cell survival (Li et al., 2002).

The overall similarity of the $\mathrm{rrol}^{-}$and $\mathrm{Dg}^{-}$phenotypes suggests that the two proteins act in the same 'polarity pathway'. In support of this view is our finding that, in trol clones, $\mathrm{Dg}$ is frequently lost from the basal-cell membrane (Fig. 4A,B). This effect seems to be specific because: (1) Dg is unaffected by the lack of Lam A (Fig. 4L); and (2) $\beta P S$ remains localized in the basal membranes of trol mutant cells that have lost Dg (Fig. 4E-G). Pcan could stabilize Dg at the basal cell surface, either by direct binding or indirectly through interaction with other cell-matrix or cellsurface proteins. Recent findings suggested a trimolecular complex of Pcan, Lam and Dg (Kanagawa et al., 2005). However, a role for Lam in stabilizing Dg in the FCE is unlikely, because Lam is not required for Dg localization (Fig. 4L). Our findings that Pcan domain V can be co-immunoprecipitated with $\mathrm{Dg}$, supports the view that Pcan stabilizes Dg at least in part by direct binding. These results suggest that direct interaction of the ECM molecule Pcan with the transmembrane protein Dg is required for the maintenance of follicle cell polarity.

In this context, it is interesting that mouse Dg is continuously shed from the cell surface of normal cutaneous cells by proteolytic cleavage of $\beta \mathrm{Dg}$ (Herzog et al., 2004). Cell culture studies with
Pcan- and Lam $\alpha 2$-deficient skin fibroblasts further revealed that shedding of Dg is increased by the lack of Pcan, but not by the lack of Lam $\alpha 2$ (Herzog et al., 2004). Drosophila Dg appears not to be processed into an $\alpha$ and a $\beta$ subunit (Fig. 5C) (Deng et al., 2003). The antibody used to detect Dg in rol $^{-}$cells was directed against the cytoplasmic domain (anti-Dg ${ }^{\text {cyto }}$ ), so clearly at least the intracellular domain of $\mathrm{Dg}$, and probably the whole protein, is lost from the cell membrane in these cells. One might speculate that the loss of Dg in trol clones represents an elevated turnover of $\mathrm{Dg}$, thereby altering the cell-matrix interaction and activity of Dg in the FCE, as shedding of Dg might do in the vertebrate system. In both systems, Pcan, but not Lam, could function to counteract this mechanism and to stabilize Dg at the cell membrane, but the expression pattern of Pcan and Dg makes clear that other mechanisms of stabilizing Dg expression must exist during early stages of oogenesis, when Pcan is not yet present in the ECM.

\section{A function for Dg without mucin-domain}

Glycosylation of $\mathrm{Dg}$ is widely accepted to be essential for its function, and recent results suggest an important role for $\mathrm{O}$ glycosylation in the mucin-domain for binding to Lam (Kanagawa et al., 2004) and Pcan (Kanagawa et al., 2005). To date, it is unclear whether the sugar-chains in the mucin-domain are directly involved in the interaction or whether they play a primarily structural function required for proper presentation of the ligand-binding domain. The following findings suggest that, in Drosophila, binding of Pcan and $\mathrm{Dg}$ does not require the mucin domain: first, the form of $\mathrm{Dg}$ that is expressed at the basal side of the FCE and depends on Pcan for its maintained localization does not contain the mucin-like domain (Fig. 5E,G); second, ectopic expression of Dg leads to ectopic accumulation of Lam and Pcan independent of the presence of the mucin domain (Fig. 5H-J); and third, one single band of $\sim 120 \mathrm{kDa}$ was detected in embryonic protein extracts in overlay binding assays with PcanV (M. Friedrich and R. Timpl, unpublished). The size of this band corresponds to the size of the two Dg forms Dg-A and Dg$\mathrm{B}$, which lack the mucin-domain. Our results suggest that the mucin- 

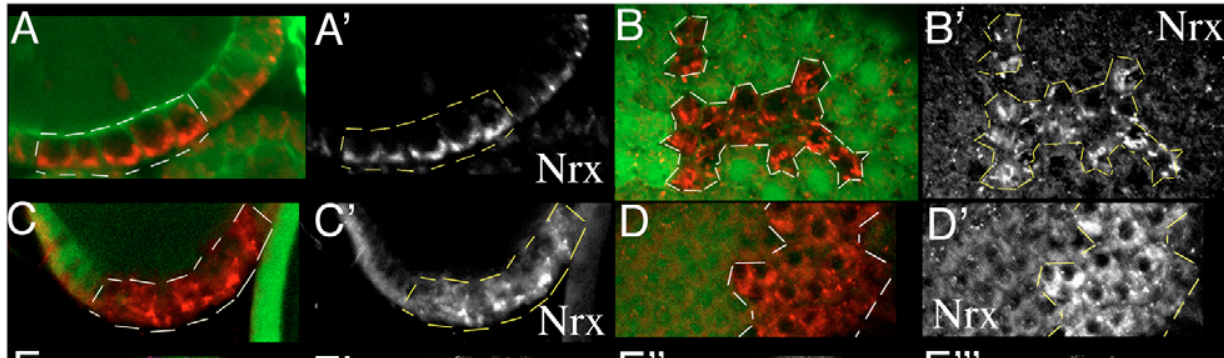

NrX

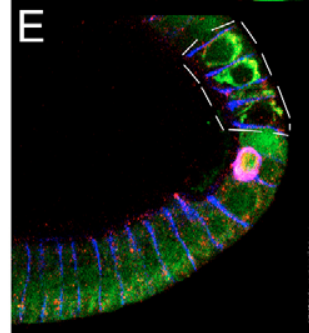

E'
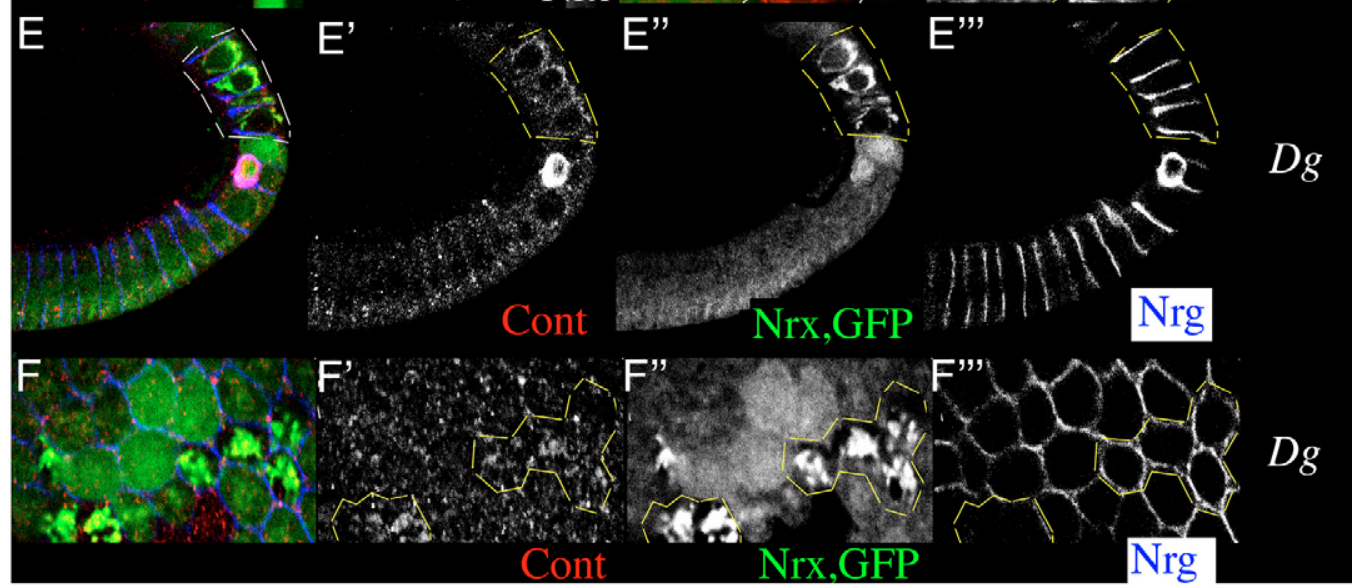

domain plays a structural role that might not be required in the specific surroundings of the FCE. Another possibility is that presence or absence of the mucin-like domain might regulate binding affinity and/or selectivity.

To our knowledge, this study is the first demonstrating a function for a Dg splicing variant lacking the mucin-like domain. It will be interesting to find out whether different Dg forms carry out different functions.

\section{Dg promotes differentiation of the basolateral membrane domain}

Contact with the ECM is important for polarization of several epithelia, including the vertebrate kidney epithelium (Eaton and Simons, 1995) and the Drosophila midgut (Yarnitzky and Volk, 1995), dorsal vessel (Haag et al., 1999) and follicular epithelia (Tanentzapf et al., 2000). In Madin-Darby canine kidney (MDCK) cells, contact with the ECM results in the formation of a basal membrane domain and in long-range effects on the differentiation of the non-basal domain (Vega-Salas et al., 1987). Similar longrange effects of ECM contact during the establishment of polarity have been observed in the Drosophila FCE (Tanentzapf et al., 2000).

Our results suggest that, after the initial polarization, ECM-cell contact mediated by Pcan and Dg plays a role in the maintenance of cell polarity. The expansion of Arm and the reduction of the lateral marker Dlg in $D g$ and trol clones might indicate a long-range effect of $\mathrm{Dg}$ on cell polarity. It is generally accepted that Dlg functions by preventing invasion of apical proteins and adherens-junction components into the lateral domain, suggesting that the reduction of Dlg in $D g$ and trol clones is the cause for the expansion of Arm in these clones. The molecular mechanisms underlying the effect of Dg on Dlg remain unknown, but our results show two clear short-range effects of $\mathrm{Dg}$ on the differentiation of the basal membrane domain: first, the recruitment and/or anchoring of the cytoplasmic protein Dystrophin and, second, the exclusion of the basolateral protein NrxIV.

\section{Interaction of Dg and Dys is conserved in Drosophila}

In vertebrates, the cytoplasmic tail of $\beta \mathrm{Dg}$ binds to Dys in muscle cells and its homolog Utr, in epithelial cells. Dys/Utr, in turn, connects to actin filaments of the cytoskeleton. Mutations in Dys cause a reduction of the expression of Dg in the sarcolemma (Ibraghimov-Beskrovnaya et al., 1992). In Drosophila, Dg and Dys are interdependent for their localization in the basal membrane of the FCE (Fig. 6) and in wing imaginal discs (M.S., unpublished), suggesting that the interaction between both proteins is conserved. Provided that Drosophila Dys also interacts with actin filaments, this result could explain the defects in basal actin organization that were observed in $D g$ clones (Deng et al., 2003).

In contrast to $D g$ clones, an abundant cytoplasmic localization of Dys was observed in trol clones. Further experiments are required to understand the precise molecular mechanisms underlying the observed defects in protein localization.

Our results raise the issue of whether Dys is also required for cell polarity. We observed that in Dys clones, the polarity marker Baz is clearly reduced, indicating a polarity defect in these cells (data not shown). The difference to $D g$ clones in which Baz is not affected, and trol clones, in which Baz expression is elevated, indicates that Dys might play a Dg-independent role in cell polarity and that the subcellular localization of Dys could play a role for its function.

\section{Dg and NrxIV localization}

Like Pcan and Lam, Neurexins contain several LG-like modules and have been described as putative interaction partners for Dg in the brain (Sugita et al., 2001). Our results suggest that, in the Drosophila FCE, Dg is required to exclude NrxIV from the basal membrane domain. Whether a direct interaction between Dg and NrxIV is involved in this process remains to be seen.

NrxIV is generally regarded as an integral component of pleated SJ. We were surprised by the finding that NrxIV is located basally to the region where $\mathrm{SJ}$ form, in a position that might correspond to 
the border between the lateral and basal cell membrane domains. The precise function of NrxIV during SJ development in the follicular epithelium remains to be elucidated.

In the embryo, NrxIV forms a complex with Nrg and Cont, and all three proteins are interdependent for SJ localization (FaivreSarrailh et al., 2004). The co-localization of NrxIV, Nrg and Cont in dot-like structures, and the fact that Cont co-localizes with ectopic NrxIV in $D g$ clones, suggest that molecular interactions between NrxIV, Cont and Nrg also occur in the FCE.

\section{A basal 'polarizing cue' in the FCE}

On the basis of our observations, we propose that Pcan and Dg provide a basal 'polarizing cue' required for differentiation of the basal membrane and maintenance of epithelial cell polarity in the FCE. Binding of the ECM molecule Pcan to its receptor Dg stabilizes Dg in the basal membrane. Dg is required for stabilizing Dlg at the lateral membrane, which in turn prevents apical markers and ZA components from invading the basolateral membrane domain. In addition, Dg forms a complex with Dys at the basal membrane and exerts a function in excluding NrxIV from the basal membrane. Further investigations will be required to understand the molecular mechanisms underlying the effect of $\mathrm{Dg}$ on $\mathrm{Dlg}$ localization and the roles of Dys and NrxIV in this process. Hopefully, a better understanding of the function of $\mathrm{Dg}$ in epithelial cell polarity will also shed some light on its role in cancer.

We thank H. Ruohola-Baker for starting a collaborative project on Drosophila Dystroglycan; S. da Rocha-Baez and C. Green for technical help; M. Durbeej and K. Gawlik for help with the glycoprotein extractions; Anne Thistle for reading the manuscript; $A$. Voigt for fly stocks; E. Knust, T. Volk, J. Fessler, M. Hortsch, M. Bhat and Developmental Studies Hybridoma Bank for antibodies; and the Bloomington Stock Center for fly stocks. Mubarak City for Science is gratefully acknowledged for giving leave permission to A.A.K. during his stay at Lund University. This work was supported by grants from Crafoordska Stiftelsen and Kungliga Fysiografiska Sällskapet i Lund to M.S., by Deutsche Forschungsgemeinschaft to A.W. [SFB 590, project A2; WO 584/4-2; DFG Research Center for Molecular Physiology of the Brain (CMPB)], by Scientist Development Grant from American Heart Association, by a Planning Grant and FYAP from FSU to W.M.D. and by Vetenskapsrådet (Grant 621-2003-3408) and Swedish Cancer Foundation (Grant 4714-B03-02XBB) to S.B.

\section{References}

Andac, Z., Sasaki, T., Mann, K., Brancaccio, A., Deutzmann, R. and Timpl, R. (1999). Analysis of heparin, alpha-dystroglycan and sulfatide binding to the $G$ domain of the laminin alpha1 chain by site-directed mutagenesis. J. Mol. Biol. 287, 253-264.

Baumgartner, S., Littleton, J. T., Broadie, K., Bhat, M. A., Harbecke, R. Lengyel, J. A., Chiquet-Ehrismann, R., Prokop, A. and Bellen, H. J. (1996) A Drosophila neurexin is required for septate junction and blood-nerve barrier formation and function. Cell 87, 1059-1068.

Bieber, A. J., Snow, P. M., Hortsch, M., Patel, N. H., Jacobs, J. R., Traquina, Z. R., Schilling, J. and Goodman, C. S. (1989). Drosophila neuroglian: a membe of the immunoglobulin superfamily with extensive homology to the vertebrate neural adhesion molecule L1. Cell 59, 447-460.

Brancaccio, A., Schulthess, T., Gesemann, M. and Engel, J. (1995). Electron microscopic evidence for a mucin-like region in chick muscle alpha-dystroglycan. FEBS Lett. 368, 139-142.

Cohn, R. D. and Campbell, K. P. (2000). Molecular basis of muscular dystrophies. Muscle Nerve 23, 1456-1471.

Collins, B. J., Gorelick, G. and Schneider, A. B. (2001). Dystroglycan is present in rat thyroid and rat thyroid cells and responds to thyrotropin. Endocrinology 142 3152-3162.

Costell, M., Gustafsson, E., Aszodi, A., Morgelin, M., Bloch, W., Hunziker, E., Addicks, K., Timpl, R. and Fassler, R. (1999). Perlecan maintains the integrity of cartilage and some basement membranes. J. Cell Biol. 147, 1109-1122.

Deng, W. M. and Ruohola-Baker, H. (2000). Laminin A is required for follicle cell-oocyte signaling that leads to establishment of the anterior-posterior axis in Drosophila. Curr. Biol. 10, 683-686

Deng, W. M., Althauser, C. and Ruohola-Baker, H. (2001). Notch-Delta signaling induces a transition from mitotic cell cycle to endocycle in Drosophila follicle cells. Development 128, 4737-4746.

Deng, W. M., Schneider, M., Frock, R., Castillejo-Lopez, C., Gaman, E. A.,
Baumgartner, S. and Ruohola-Baker, H. (2003). Dystroglycan is required for polarizing the epithelial cells and the oocyte in Drosophila. Development 130 173-184.

Eaton, S. and Simons, K. (1995). Apical, basal, and lateral cues for epithelial polarization. Cell $\mathbf{8 2}, 5-8$

Faivre-Sarrailh, C., Banerjee, S., Li, J., Hortsch, M., Laval, M. and Bhat, M. A. (2004). Drosophila contactin, a homolog of vertebrate contactin, is required for septate junction organization and paracellular barrier function. Development 131, 4931-4942.

Friedrich, M. V., Schneider, M., Timpl, R. and Baumgartner, S. (2000). Perlecan domain $\mathrm{V}$ of Drosophila melanogaster. Sequence, recombinant analysis and tissue expression. Eur. J. Biochem. 267, 3149-3159.

Greener, M. J. and Roberts, R. G. (2000). Conservation of components of the dystrophin complex in Drosophila. FEBS Lett. 482, 13-18.

Gutzeit, H. O., Eberhardt, W. and Gratwohl, E. (1991). Laminin and basement membrane-associated microfilaments in wild-type and mutant Drosophila ovarian follicles. J. Cell Sci. 100, 781-788

Haag, T. A., Haag, N. P., Lekven, A. C. and Hartenstein, V. (1999). The role of cell adhesion molecules in Drosophila heart morphogenesis: faint sausage, shotgun/DE-cadherin, and laminin A are required for discrete stages in heart development. Dev. Biol. 208, 56-69.

Henchcliffe, C., Garcia-Alonso, L., Tang, J. and Goodman, C. S. (1993) Genetic analysis of laminin A reveals diverse functions during morphogenesis in Drosophila. Development 118, 325-337.

Henry, M. D. and Campbell, K. P. (1999). Dystroglycan inside and out. Curr. Opin. Cell Biol. 11, 602-607.

Henry, M. D., Cohen, M. B. and Campbell, K. P. (2001). Reduced expression of dystroglycan in breast and prostate cancer. Hum. Pathol. 32, 791-795.

Herzog, C., Has, C., Franzke, C. W., Echtermeyer, F. G., Schlotzer-Schrehardt, U., Kroger, S., Gustafsson, E., Fassler, R. and Bruckner-Tuderman, L. (2004). Dystroglycan in skin and cutaneous cells: beta-subunit is shed from the cell surface. J. Invest. Dermatol. 122, 1372-1380.

Hohenester, E., Tisi, D., Talts, J. F. and Timpl, R. (1999). The crystal structure of a laminin G-like module reveals the molecular basis of alpha-dystroglycan binding to laminins, perlecan, and agrin. Mol. Cell 4, 783-792.

Ibraghimov-Beskrovnaya, O., Ervasti, J. M., Leveille, C. J., Slaughter, C. A., Sernett, S. W. and Campbell, K. P. (1992). Primary structure of dystrophinassociated glycoproteins linking dystrophin to the extracellular matrix. Nature 355, 696-702

lozzo, R. V. (1994). Perlecan: a gem of a proteoglycan. Matrix Biol. 14, 203-208. Julenius, K., Molgaard, A., Gupta, R. and Brunak, S. (2005). Prediction, conservation analysis, and structural characterization of mammalian mucin-type O-glycosylation sites. Glycobiology 15, 153-164.

Kanagawa, M., Saito, F., Kunz, S., Yoshida-Moriguchi, T., Barresi, R. Kobayashi, Y. M., Muschler, J., Dumanski, J. P., Michele, D. E., Oldstone, M. B. et al. (2004). Molecular recognition by LARGE is essential for expression of functional dystroglycan. Cell 117, 953-964

Kanagawa, M., Michele, D. E., Satz, J. S., Barresi, R., Kusano, H., Sasaki, T. Timpl, R., Henry, M. D. and Campbell, K. P. (2005). Disruption of perlecan binding and matrix assembly by post-translational or genetic disruption of dystroglycan function. FEBS Lett. 579, 4792-4796.

Klebes, A. and Knust, E. (2000). A conserved motif in Crumbs is required for Ecadherin localisation and zonula adherens formation in Drosophila. Curr. Biol. 10, 76-85.

Kumagai, C., Kadowaki, T. and Kitagawa, Y. (1997). Disulfide-bonding between Drosophila laminin beta and gamma chains is essential for alpha chain to form alpha betagamma trimer. FEBS Lett. 412, 211-216.

Li, S., Harrison, D., Carbonetto, S., Fassler, R., Smyth, N., Edgar, D. and Yurchenco, P. D. (2002). Matrix assembly, regulation, and survival functions of laminin and its receptors in embryonic stem cell differentiation. J. Cell Biol. 157, 1279-1290

Li, S., Edgar, D., Fassler, R., Wadsworth, W. and Yurchenco, P. D. (2003). The role of laminin in embryonic cell polarization and tissue organization. Dev. Cell $\mathbf{4}$, 613-624.

Margolis, J. and Spradling, A. (1995). Identification and behavior of epithelial stem cells in the Drosophila ovary. Development 121, 3797-3807.

Muller, H. A. (2000). Genetic control of epithelial cell polarity: lessons from Drosophila. Dev. Dyn. 218, 52-67.

Muschler, J., Levy, D., Boudreau, R., Henry, M., Campbell, K. and Bissell, M. J. (2002). A role for dystroglycan in epithelial polarization: loss of function in breast tumor cells. Cancer Res. 62, 7102-7109.

Peng, H. B., Xie, H., Rossi, S. G. and Rotundo, R. L. (1999). Acetylcholinesterase clustering at the neuromuscular junction involves perlecan and dystroglycan. J. Cell Biol. 145, 911-921.

Pignoni, F. and Zipursky, S. L. (1997). Induction of Drosophila eye development by decapentaplegic. Development 124, 271-278.

Sciandra, F., Schneider, M., Giardina, B., Baumgartner, S., Petrucci, T. C. and Brancaccio, A. (2001). Identification of the beta-dystroglycan binding epitope within the C-terminal region of alpha-dystroglycan. Eur. J. Biochem. 268, 45904597. 
Sgambato, A. and Brancaccio, A. (2005). The dystroglycan complex: from biology to cancer. J. Cell Physiol. 205, 163-169.

Sgambato, A., Migaldi, M., Montanari, M., Camerini, A., Brancaccio, A., Rossi, G., Cangiano, R., Losasso, C., Capelli, G., Trentini, G. P. et al. (2003). Dystroglycan expression is frequently reduced in human breast and colon cancers and is associated with tumor progression. Am. J. Pathol. 162 849-860.

Smalheiser, N. R. and Kim, E. (1995). Purification of cranin, a laminin binding membrane protein. Identity with dystroglycan and reassessment of its carbohydrate moieties. J. Biol. Chem. 270, 15425-15433.

Sugita, S., Saito, F., Tang, J., Satz, J., Campbell, K. and Sudhof, T. C. (2001). A stoichiometric complex of neurexins and dystroglycan in brain. J. Cell Biol. 154 435-445.

Talts, J. F., Andac, Z., Gohring, W., Brancaccio, A. and Timpl, R. (1999). Binding of the $\mathrm{G}$ domains of laminin alpha1 and alpha2 chains and perlecan to heparin, sulfatides, alpha-dystroglycan and several extracellular matrix proteins. $E M B O$ J. 18, 863-870.

Tanentzapf, G., Smith, C., McGlade, J. and Tepass, U. (2000). Apical, lateral, and basal polarization cues contribute to the development of the follicular epithelium during Drosophila oogenesis. J. Cell Biol. 151, 891-904.
Vega-Salas, D. E., Salas, P. J., Gundersen, D. and Rodriguez-Boulan, E. (1987). Formation of the apical pole of epithelial (Madin-Darby canine kidney) cells: polarity of an apical protein is independent of tight junctions while segregation of a basolateral marker requires cell-cell interactions. J. Cell Biol. 104, 905-916.

Voigt, A., Pflanz, R., Schafer, U. and Jackle, H. (2002). Perlecan participates in proliferation activation of quiescent Drosophila neuroblasts. Dev. Dyn. 224, 403412.

Wei, J., Hortsch, M. and Goode, S. (2004). Neuroglian stabilizes epithelial structure during Drosophila oogenesis. Dev. Dyn. 230, 800-808.

Winder, S. J. (2001). The complexities of dystroglycan. Trends Biochem. Sci. 26 118-124.

Wodarz, A., Ramrath, A., Grimm, A. and Knust, E. (2000). Drosophila atypical protein kinase $C$ associates with Bazooka and controls polarity of epithelia and neuroblasts. J. Cell Biol. 150, 1361-1374.

Woods, D. F. and Bryant, P. J. (1991). The discs-large tumor suppressor gene of Drosophila encodes a guanylate kinase homolog localized at septate junctions. Cell 66, 451-464.

Yarnitzky, T. and Volk, T. (1995). Laminin is required for heart, somatic muscles, and gut development in the Drosophila embryo. Dev. Biol. 169, 609-618. 